\title{
Schwangerschafts- und Geburtskomplikationen - ihr Stellenwert in der Entstehung schizophrener Psychosen
}

\author{
G. Stöber, E. Franzek, H. Beckmann
}

Psychiatrische Klinik und Poliklinik der Universität Würzburg (Direktor: Prof. Dr. H. Beckmann)

\begin{abstract}
Birth and Pregnancy Complications Their Role in the Development of Leonhard's Unsystematic and Systematic Schizophrenias
\end{abstract}

In a retrospective study of 80 chronic DSM III-R schizophrenics and 80 controls, the occurrence of obstetric complications (OCs) into the development of chronic schizophrenias was investigated using Leonhard's distinction in systematic schizophrenia (no obvious familial loading) and unsystematic schizophrenia (mainly genetically determined according to Leonhard). The Lewis \& Murray and Fuchs scales were used for evaluation. In both scales, unsystematic schizophrenias did not differ from controls, but those with OCs were significantly $(p<0.01)$ earlier hospitalized (20.5 years) than those without OCs (25.6 years). Systematic schizophrenics had an increased frequency, severity and total score of OCs compared to controls in the Fuchs scale $(\mathrm{p}<0.01)$. Likewise, in the Lewis \& Murray scale systematic schizophrenia showed an increased presence of OCs compared to controls $(p<0.05)$ and to unsystematic schizophrenia $(p<0.1)$. Systematic schizophrenias were significantly allocated to maternal infectious diseases during mid-gestation. Patients with maternal infections showed more additional OCs than those without ( $p<0.05 ;$ Lewis \& Murray scale). In systematic schizophrenia, a history of OC was not associated with an early onset of the disease. In the genetic determined schizophrenias prenatal and perinatal disturbances lead to an early onset of the disease, however, in systematic schizophrenias they seem to be of causal importance for the development of the disease.

\section{Einleitung}

Ob Schwangerschafts- und vor allem Geburtskomplikationen durch hypoxisch-ischämische zerebrale Schädigungen eine erhöhte Vulnerabilität gegenüber schizophrenen Psychosen hervorrufen und so mit zum Entstehen dieser Erkrankungen beitragen, wird kontrovers diskutiert (Goodman, 1988; McNeil, 1987).

Fortschr. Neurol. Psychiat. 61 (1993) 329-337

(1) Georg Thieme Verlag Stuttgart - New York

\section{Zusammenfassung}

Auf der Grundlage von Leonhards Unterteilung in systematische Schizophrenien (niedriges genetisches Risiko) und unsystematische Schizophrenien (nach Leonhards Befunden hauptsächlich genetisch determiniert) wurden in einer retrospektiven Studie bei 80 Patienten mit chronischen DSM III-R Schizophrenien und 80 Kontrollen die Häufigkeit von Schwangerschafts- und Geburtskomplikationen untersucht. Zur Auswertung wurden die Skalen von Lewis \& Murray sowie von Fuchs verwandt. Unsystematische Schizophrenien unterschieden sich in beiden Skalen nicht von den Kontrollen. Diejenigen mit Komplikationen wurden jedoch signifikant früher als diejenigen ohne Komplikationen ersthospitalisiert $(p<0,01)$. Bei systematischen Schizophrenen waren in der Skala von Fuchs Häufigkeit, Schweregrad sowie der Summenwert der Komplikationen gegenüber den Kontrollen erhöht $(p<0,01)$. Auch in der Skala von Lewis \& Murray traten häufiger obstetrische Komplikationen auf als bei Kontrollen $(p<0,05)$ und unsystematischen Schizophrenen $(p<0,1)$. Systematische Schizophrenien waren auch assoziert mit Schwangerschaftsinfektionen im zweiten Trimenon. Mütter mit Schwangerschaftsinfektionen zeigten gehäuft weitere perinatala Komplikationen $(p<0,05)$. Geburtskomplikationerı hatten bei systematischen Schizophrenen jedoch keinen Ein. fluß auf den Zeitpunkt des Krankheitsbeginns. Während prä-und perinatale Störungen bei genetisch determinierten Schizophrenien lediglich einen frühen Krankheitsbeginn bewirken, scheinen sie bei den systematischen Schizophrenien von ursächlicher Bedeutung für die Krankheitsentstehung zu sein.

Pollack et al. (1966) wiesen erstmals darauf hin, daß schizophrene Patienten mehr geburtshilfliche Komplikationen als ihre gesunden Geschwister hatten. Dies konnte von mehreren Gruppen repliziert werden (Tab. 1 a). Eagles et al. (1990) fanden bei 27 Geschwisterpaaren, daß die an einer Schizophrenie Erkrankten gehäuft schwere Komplikationen erlitten hatten. Diese Unterschiede bestätigten sich auch in Familien mit mehreren psychosekranken Geschwistern. Diese Geschwisterpaare hatten deutlich mehr pathologische Schwangerschaften und Geburten durchgemacht als ihre psychisch gesund gebliebenen Geschwister (De Lisi et al., 1987, 1988). 
Tab. 1 Häufigkeit von Schwangerschafts- und Geburtskomplikationen bei Patienten mit schizophrenen Psychosen.

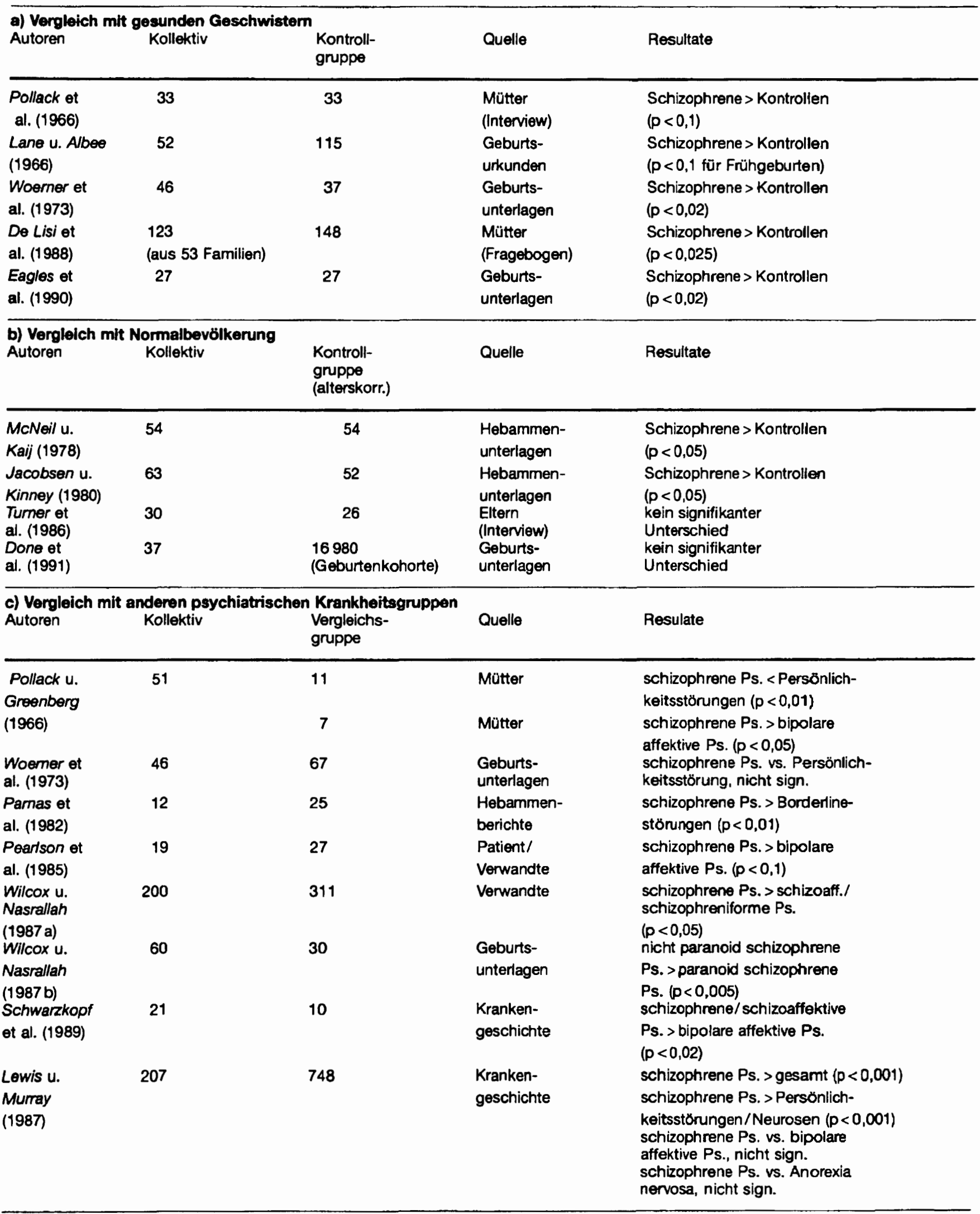


Verglichen mit der Normalbevölkerung wiesen die Geburten Schizophrener häufiger prä- und neonatale sowie intrapartale Zwischenfälle auf (Tab. 1 b). McNeil u. Kaij (1978) fanden bei eng definierten „Prozeßschizophrenen“ im Vergleich zu alters- und geschlechtskorrelierten Kontrollen eine signifikant erhöhte Zahl von geburtshilflichen Komplikationen (vor allem Weheninsuffizienzen) und von postpartalen Auffälligkeiten. Asphyxien und Zeichen der Frühreife waren bei den Neugeborenen gehäuft. Bei Patienten mit akuten schizophreniformen Psychosen kamen dagegen Geburts- und Schwangerschaftskomplikationen nicht wesentlich vermehrt vor (McNeil, 1988).

Die Häufigkeit von perinatalen Störungen untersuchten Jacobson u. Kety (1980) bei schizophrenen Patienten mit einem größtenteils gutartigen Krankheitsverlauf mit Remissionen (Kety et al., 1968). Gegenüber den alters- und geschlechtskorrelierten Kontrollen fanden sich in der Patientengruppe signifikant mehr und schwercrc Komplikationen. Besonders oft waren protrahierte Geburtsverläufe vorgekommen. Der soziale Status der Eltern hatte auf die Häufigkeit obstetrischer Komplikationen keinen Einfluß. Turner et al. (1986) fanden in einer Gruppe von 30 DSM-III-Schizophrenen, die innerhalb der ersten zwei Krankheitsjahre untersucht wurden, und deren Verlaufsprognose ungeklärt blieb, Schwangerschafts- und Geburtskomplikationen vergleichbar häufig wie bei alterskorrelierten Gesunden. Done et al. (1991) reanalysierten die Daten einer Geburtenkohorte von 16980 Kindern, die 1958 mit der Fragestellung untersucht worden waren, welche prä- und perinatalen Komplikationen die Säuglingssterblichkeit erhöhen. Bei 37 Patienten, die später mit schizophrenen Psychosen hospitalisiert wurden, waren Schwangerschafts- und Geburtskomplikationen, die in der gesamten Geburtenkohorte die Säuglingssterblichkeit erhöht hatten, nur in Richtung eines statistischen Trends vermehrt vorgekommen.

Prä- und perinatale Auffälligkeiten scheinen bei verschiedenen psychiatrischen Erkrankungen unterschiedlich hoch zu sein (Tab. 1 c). Nach den Befunden von Wilcox u. Nasrallah (1987a) hatten schizophrene Patienten signifikant mehr Schwangerschafts- und Geburtszwischenfälle als Patienten, die an einer schizophreniformen oder schizoaffektiven Psychose litten. Innerhalb der Schizophrenen fanden diese Autoren (Wilcax u. Nasrallah, 1987 b) ein Überwiegen der Komplikationen bei Patienten mit einem nicht paranoiden Typus. Patienten mit affektiven Psychosen und Patienten mit Anorexia nervosa wiesen in der Untersuchung von Lewis u. Murray (1987) eine ähnlich hohe Zahl von Schwangerschafts- und geburtshilflichen Komplikationen auf wie Schizophrene. An bipolaren affektiven Psychosen Erkrankte können aber auch wesentlich seltener Komplikationen als Patienten mit Schizophrenien erleiden (Pollack et al., 1966; Pearlson et al., 1985; Schwarzkopf et al., 1988). Ähnlich divergierendc Bcfunde ergaben sich bei einem Vergleich mit Patienten, bei denen Neurosen oder Persönlichkeitsstörungen bestanden (Tab.1c). Während Pollack et al. (1966) perinatale Störungen überwicgend bei Patienten mit Persönlichkeitsakzentuierungen fand, waren für Woerner et al. (1973) keine Unterschiede evident und Lewis u. Murray (1987) fanden neuerdings bei diesen Patienten wesentlich seltener Geburts- und Schwangerschaftskomplikationen als bei Schizophrenen.

Nachdem Rieder et al. (1975) darauf hingewiesen hatten, daß in der Nachkommenschaft schizophrener Eltern die Zahl der Totgeburten und die Säuglingssterblichkeit überproportional hoch sei, wurde gemutmaßt, die Rate an Schwangerschafts- und Geburtskomplikationen sei bei Kindern von schizophrenen Eltern allgemein erhöht. Wie McNeil und Kaij (1978) in einer Übersichtsarbeit zeigen konnten, und auch neuere Untersuchungen (Marcus et al., 1981; McNeil, 1988) bestätigten, haben Kinder schizophrener Mütter nicht generell ein höheres Risiko für Geburtskomplikationen. In der dänischen "high-risk"-Studie (Parnas et al., 1982) hatten die Kinder von schizophrenen Müttern, die später selbst an einer Schizophrenie erkrankten, eine nur leicht erhöhte Rate an Komplikationen gegenüber denjenigen, die psychisch gesund blieben. Diejenigen mit einer schizotypischen Persönlichkeit hatten interessanterweise signifikant weniger Komplikationen als die Schizophrenen durchgemacht.

Pollack und Greenberg (1966) hatten zeigen können, $\mathrm{da}$ pathologische Schwangerschaften und Geburten zu einer frühen Krankheitsmanifestation führen. In ihrer Untersuchung waren nur $14 \%$ der schizophrenen Patienten ohne Kornplikationen, hingegen $89 \%$ von denen mit schweren perinatalen Komplikationen vor dem 18. Lebensjahr hospitalisiert worden. Pearlson et al. (1985), Delisi et al. (1987), Owen et al. (1988) und $O^{\prime}$ Callaghan et al. (1990a) gingen mit diesen Befunden konform. In den Kollektiven von Wilcox und Nasrallah (1987 a) und Reddy et al. (1990) beeinflußten Schwangerschafts- und Geburtskomplikationen den Zeitpunkt des Krankheitsbeginnes hingegen nicht.

Auch blieb umstritten, ob sich eine genetische Disposition zu schizophrenen Psychosen und obstetrische Komplikationen in ihren Wirkungen ergänzen. Pearlson et al. (1985), Nimgaonkar et al. (1988) und Reddy et al. (1990) fanden keinen Unterschied zwischen Patienten mit einer positiven oder einer negativen Familienanamnese für schizophrene Psychosen. Patienten mit leerer Familienanamnese hatten hingegen signifikant gehäuft prä- und perinatale Komplikationen in den Untersuchungen von Wilcox und Nasrallah (1987a), Schwarzkopf et al. (1990) und $O^{\prime}$ Callaghan et al. (1990a). Sie nahmen an, daß Störungen in der Schwangerschaft, während der Geburt und während der Neonatalzeit von genetischen Einflüssen unabhängige Faktoren sind, die zum Entstehen schizophrener Psychosen beitragen.

Die psychopathologische Forschung von Wernicke und Kleist weiterführend, gelang Leonhard (1986) eine differenzierte Aufteilung der endogenen Psychosen nach ihrem Erscheinungsbild und ihrer Prognose. Nach Absonderung der gutartig verlaufenden zykloiden Psychosen (Leonhand, 1972), bei denen der akuten Erkrankung stets eine Vollremission folgt, unterteilte Leonhard die Schizophrenien in zwei klar voneinander zu trennende Hauptgruppen: in unsystematische und in systematische Schizophrenien. Die psychopathologischen Erscheinungsbilder und die Verlaufsprognosen sind bei beiden Gruppen völlig unterschiedlich. Die unsystematischen Formen (affektvolle Paraphrenie, periodische Katatonie und Kataphasie) gehen über meist mehrere, voneinander abgrenzbare Krankheitsschübe mit akut produktiver und vielgestaltiger Symptomatik in unterschiedlich schwer ausgeprägte Residuen über. Bei diesen Krankheitsformen fand Leonhard (1975) eine hohe familiäre Belastung mit gleichartigen Psychosen. Hingegen zeigen die systematischen Schizophrenien eng umschriebene Syndrome, die von Leonhard (1986) höchst subtil beschrieben und voneinander abgegrenzt wurden. Die Krankheitsfor- 
Tab. 2 Schwangerschafts- und Geburtskomplikationen bei Schizophrenen in Abhăngigkeit von einer positiven oder negativen Familienanamnese.

\begin{tabular}{|c|c|c|c|}
\hline Autoren & $\begin{array}{l}\text { Patienten mit negativer } \\
\text { Familienanamnese } \\
\text { (Rate der Kompli- } \\
\text { kationen in \%) }\end{array}$ & $\begin{array}{l}\text { Patienten mit positiver } \\
\text { Familienanamnese } \\
\text { (Rate der Kompli- } \\
\text { kationen in \%) }\end{array}$ & Resultate \\
\hline $\begin{array}{l}\text { Peartson et } \\
\text { al. (1985) }\end{array}$ & $13(23 \%)$ & $6(17 \%)$ & nicht signifikant \\
\hline $\begin{array}{l}\text { Nimgaonkar } \\
\text { et al. (1988) }\end{array}$ & 30 (nicht berichtet) & 18 (nicht berichtet) & nicht signifikant \\
\hline $\begin{array}{l}\text { Reddy et } \\
\text { al. (1990) }\end{array}$ & $22(50 \%)$ & $20(50 \%)$ & nicht signifikant \\
\hline Wilcox u. & $245(25 \%)$ & $266(9 \%)$ & negativ > positiv \\
\hline Nasrallah (1987 a) & & & $(p<0,025)$ \\
\hline $\begin{array}{l}\text { Schwarzkopf } \\
\text { et al. (1989) }\end{array}$ & 19 (nicht berichtet) & 9 (nicht berichtet) & $\begin{array}{l}\text { negativ }>\text { positiv } \\
(p<0,013)\end{array}$ \\
\hline $\begin{array}{l}\text { O'Callaghan } \\
\text { et al. (1990) }\end{array}$ & $29(45 \%)$ & $16(13 \%)$ & $\begin{array}{l}\text { negativ }>\text { positiv } \\
(p<0,065)\end{array}$ \\
\hline
\end{tabular}

men der systematischen Katatonien, Hebephrenien und $\mathrm{Pa}$ raphrenien sind auch heute noch trotz psychopharmakologischer Behandlung in ihren irreversiblen Endzuständen zu beobachten (Astrup, 1979; Stöber et al., 1993a) und reliabel diagnostizierbar (Franzek u. Beckmann, 1991). Diese Formen der Schizophrenien lassen nach Leonhards Befunden (1986) eine positive Familienanamnese meist vermissen. Aus der klinischen Differenzierung der schizophrenen Krankheitsformen ergab sich so auch eine verschiedenartige genetische Disposition. Den unsystematischen, nach Leonhards Befunden hereditären Schizophrenien sind die systematischen Schizophrenien gegenüberzustellen. Diese zeigen nach seinen Ergebnissen keine Erblichkeit. In ihrer Entstehung nahm Leonhard (1979) psychosoziale und Umwelteinflüsse als ursächliche Faktoren an.

In einer ersten Untersuchungsreihe (Stöber et al., 1992, 1993 b) hatten wir bei systematischen Schizophrenien gehäuft Schwangerschaftsinfektionen der Mütter sowie weitere prä- und perinatale Komplikationen gefunden. Im erweiterten Untersuchungsgut konnten wir bei Müttern von Patienten mit systematischen Schizophrenien signifikant gehäuft Schwangerschaftsinfektionen im zweiten Trimenon und insbesondere im 5. Schwangerschaftsmonat im Vergleich zu Kontrollen nachweisen (Stöber et al., 1993 c). Bei den unsystematischen Schizophrenien waren kaum Infektionen aufgetreten.

Hier soll im erweiterten Kollektiv den Auswirkungen von weiteren Schwangerschafts- und Geburtskomplikationen auf die Entwicklung schizophrener Psychosen unter dem Blickwinkel einer differenzierten Psychopathologie nachgegangen werden.

\section{Patienten und Methodik}

In die Studie aufgenommen wurden Patienten der Psychiatrischen Universitätsklinik Würzburg und des Krankenhauses für Psychiatrie und Neurologie in Lohr a.M. Die Patienten mußten die diagnostischen Kriterien einer chronischen Schizophrenie nach dem DSM-IIl-R (1987) und die restriktiven diagnostischen Kriterien für eine unsystematische oder eine systematische Schizophrenie nach der Leonhard-Klassifikation erfüllen (im Detail: Stöber et al., 1992, 1993 c). Bisher wurden 80 Patienten (21 Frauen, 59 Männer), deren Mütter noch lebten, in die Untersuchung aufgenommen. Zum Zeitpunkt der Untersuchung waren die Patienten durchschnittlich 35,3 ( $\pm 8,83$ ) Jahre alt. Sie waren im Alter von $23,5( \pm 6,95)$ Jahren erstmals hospitalisiert
Tab. 3 Schwangerschafts- und Geburtskomplikationen be Schizophrenen und das Alter bei der Ersthospitalisierung.

\begin{tabular}{|c|c|c|c|c|}
\hline Autoren & Kollektiv & $\begin{array}{l}\text { Alter } \\
\text { Ersth } \\
\text { Komf } \\
\text { mit }\end{array}$ & $\begin{array}{l}\text { lisierung } \\
\text { nen } \\
\text { ohne }\end{array}$ & Signifikanz \\
\hline $\begin{array}{l}\text { Pollack u. } \\
\text { Greenberg (1966) }\end{array}$ & 51 & 18,4 & 22,9 & $p<0,01$ \\
\hline $\begin{array}{l}\text { Pearlson et } \\
\text { al. (1985) }\end{array}$ & 19 & 17,3 & 22,5 & $p<0,1$ \\
\hline $\begin{array}{l}\text { Wilcox u. } \\
\text { Nasrallah (1987 a) }\end{array}$ & 200 & nicht & itet & nicht signifikant \\
\hline $\begin{array}{l}\text { Reddy et } \\
\text { al. }(1990)\end{array}$ & 44 & 19,4 & 18,6 & nicht signifikant \\
\hline $\begin{array}{l}\text { Owen et al. } \\
(1988)\end{array}$ & 110 & 18,6 & 22,5 & $p<0,05$ \\
\hline $\begin{array}{l}\text { O'Callaghan } \\
\text { ot al. (1992) }\end{array}$ & 65 & 20,1 & 24,8 & $p<0,02$ \\
\hline
\end{tabular}

worden. lhre Erkrankung bestand seit 16,6 $( \pm 9,25)$ Jahren. Das durchschnittliche Alter der Mütter betrug zum Zeitpunkt des Interviews 64,3 $( \pm 9,65)$ Jahre. Die Patienten wurden - unabhängig voneinander - von H. B. und E. F, untersucht. Diese erzielten in einer kürzlich veröffentlichten Studie in der Diagnostik der endogenen Psychosen nach Leonhard einen hohen Übereinstimmungskoeffizienten (Cohens kappa: 0,88) (Franzek u. Beckmann, 1992).

Bei 36 Patienten (8 Frauen, 28 Männer) wurden systematische Schizophrenien diagnostiziert. Sie waren mit durchschnittlich 23,6 ( $\pm 7,98)$ Jahren erstmals hospitalisiert worden. Zum Zeitpunk der Untersuchung waren sie $34,8( \pm 9,44)$ Jahre alt. Unsystematische Schizophrenien nach Leonhard lagen bei 44 Patienten (13 Frauen, 3 I Männer) vor. Sie waren zum Untersuchungszeitraum 35,7 ( $\pm 8,39$ ) Jahre alt. Diese Patienten waren mit $23,0( \pm 6,06)$ Jahren erstmals in einer psychiatrischen Klinik behandelt worden. Der Median für das Alter bei der Ersthospitalisierung betrug für die unsystematischen 21,5 Jahtre und für die systematischen Schizophrenen 23 Jahre. Als Vergleichsgruppe dienten 80 alterskorrelierte, psychisch und physisch gesunde Probanden, von denen keiner einen schizophrenen Verwandien ersten oder zweiten Grades hatte. Alle Kontrollpersonen hatten zum Zeitpunkt des Interviews eine abgeschlossene Berufsbildung und waren berufstätig. Zwischen den diagnostischen Untergruppen ergaben sich im t-Test keine statistisch signifikanten Unterschiede für die biographischen und klinischen Eckdaten der Patienten und für das Alter der Mütter zum Zeitpunkt des Interviews. 
Tab. 4 Unsystematische und systematische Schizophrenlen nach Leonhard: Haufigkeit und Schweregrad sowie gewichtete Gesamtsumme der Schwangerschafts- und Geburtskomplikationen im Vergleich zu gesunden Kontrollen (Fuchs-Skala 1982).

\begin{tabular}{llll}
\hline $\begin{array}{l}\text { Schwangerschafts- Kontrollen } \\
\text { und Geburts- } \\
\text { komplikationen }\end{array}$ & $\begin{array}{l}\text { unsystematische } \\
\text { Schizophrenien } \\
(n=80)\end{array}$ & $\begin{array}{l}\text { systematische } \\
\text { Schizophrenien } \\
(n=36)\end{array}$ \\
\hline $\begin{array}{l}\text { Häufigkeit } \\
\text { Schweregrad }\end{array}$ & $1,01 \pm 1,281,25 \pm 1,38$ & $1,97 \pm 1,82^{*}$ \\
$\begin{array}{l}\text { gewichtete } \\
\text { Gesamtsumme }\end{array}$ & $1,26 \pm 1,521,71 \pm 1,76$ & $1,92 \pm 1,65^{*}$ \\
& $2,03 \pm 2,963,00 \pm 3,56$ & $3,94 \pm 4,29^{*}$
\end{tabular}

- Bei systematisch Schizophrenen besteht gegenüber unsystematischen Formen eine erhohte Frequenz an Komplikationen $(p<0,05 ; t=2,011$ ) und gegenüber Kontrollen sind Häutigkeit $(p<0,01 ; t=3,256)$, Schweregrad $(p<0,05 ; t=2,086)$ sowie gewichtete Gesamtsumme $(p<0,01$; $t=2,792$ ) ertoht.

Mehrere Untersuchungsgruppen (Joffe et al., 1985; Tilley et al., 1986; $O^{\prime}$ Callaghan et al., 1990 b) konnten eindrucksvoll belegen, daß Mütter auch noch nach vielen Jahren Schwangerschaftsund Geburtskomplikationen in guter Übereinstimmung mit den ursprünglichen ärztlichen Aufzeichnungen erinnern.

Dies berechtigte uns zu einer retrospektiven Untersuchungsmethode. Wir führten bei den Müttern der chronisch Schizophrenen und bei den Müttern von gesunden Kontrolipersonen ein strukturiertes Interview durch, um die Häufigkeit und Spezifität von verschiedenen Schwangerschafts- und Geburtskomplikationen und von Störungen der postnatalen Entwicklung bei schizophrenen Patienten zu erfassen. Die Diagnosen und die psychiatrische Familienanamnese der Patienten waren dem Untersucher (G. S.) nicht bekannt und durften während des Interviews nicht erhoben werden. Die Angaben darüber waren erst nach Abschluß der Untersuchungsreihe für den Interviewer zugänglich. Mit den Müttern der Patienten wurde ein persönlicher Untersuchungstermin vereinbart. Jedes Interview nahm ungefähr $2 \mathrm{~h}$ in Anspruch. Alle Fragen zu Schwangerschafts- und Geburtskomptikationen wurden persönlich gestellt. Wichtige Ereignisse wurden vom Lntersucher schriftlich genau festgehalten.

Anhand zweier Skalen wurden die berichteten Komplikationen gewertet. In der "Obstetric Complication Scale" von Lewis und Murray (1987) werden 15 verschiedene Arten von Komplikationen erfaßt und mit 0 (abwesend), 1 (wahrscheinlich anwesend) und 2 (anwesend) gewertet. Es werden vier Schwangerschafts-, neun Geburtskomplikationen sowie zwei postpartale Komplikationen aufgeführt. In der "Severity weight allocation sćale for specific complications" (Parmas et al., 1982) werden 24 verschiedene Arten von Schwangerschaftsund Geburtszwischenfällen erfaßt. Sie erhalten einen Wert zwischen 0 und 4, der dem Schweregrad Rechnung trägt. In dieser Skala können Komplikationen nach ihrer Hāufigkeit und nach ihrem Schweregrad getrennt voneinander untersucht und zu einer gewichteten Gesamtsum. me addiert werden. In der von Fuchs entwickeiten Skala werden Schwangerschafts- und Geburtskomplikationen als somatische Abweichungen von einem zu erwartenden, optimalen Verlauf der Schwangerschaft, der Entbindung und der Neonatalzeit aufgefaßt. Die Skala von Lewis und Murray (1987) umfaßt Störungen der prä- und perinatalen Zeit, die das Risiko der fetalen Mortalität erhöhen. Methodische Vorgaben und Validität beider Skalen wurden von Molfese et al. (1985) und Lewis et al. (1989) eingehend diskutiert.

Zur statistischen Analyse wurden der t-Test sowie der Chi 2 -Test verwandt.

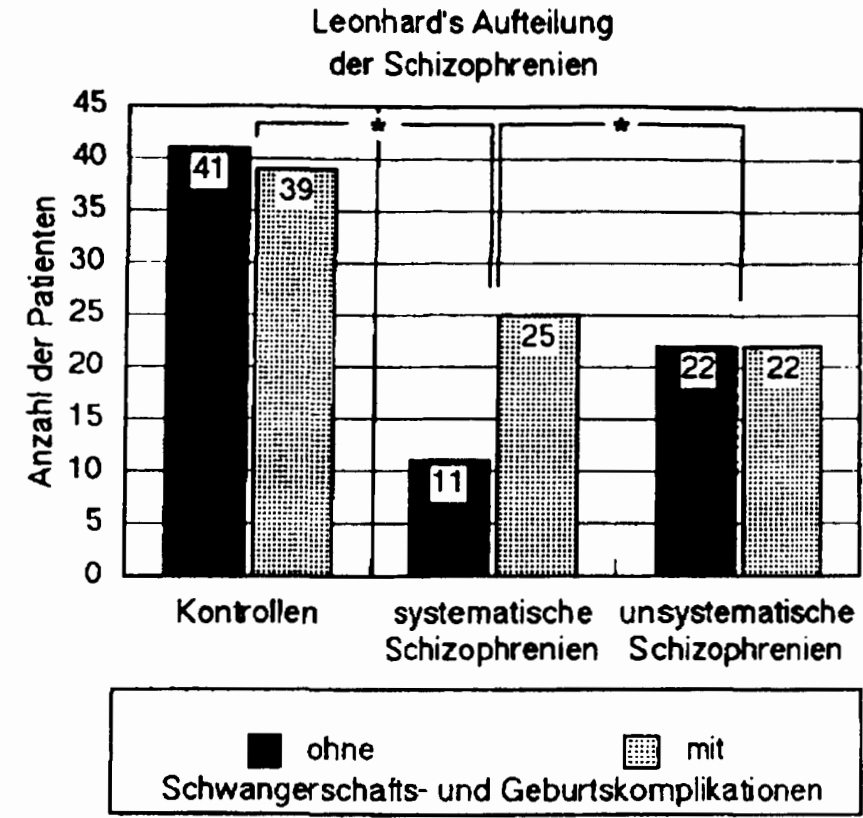

Abb. 1 Bei systematischen, nicht hereditären Schizophrenien sind pathologische Schwangerschaften und Geburten häufiger als bei Kontrollen $\left(\mathrm{p}<0,05 ; \mathrm{df}=1\right.$; $\mathrm{Chi}_{2}=4,29$ ) und bei unsystematischen, genetisch determinierten Schizophrenien $(p<0,1 ; d f=1$; $\mathrm{Chi}_{2}=3,09 ;$ Skala von Lewis und Murray).

\section{Resultate}

Skala zur Erfassung spezifischer Schwangerschafts- und Geburtskomplikationen (Fuchs-Skala; Tab. 4)

53 der 80 schizophrenen Patienten (66\%) und 44 der 80 Kontrollpersonen (55\%) erlitten nach dieser Skala Komplikationen.

Patienten mit unsystematischen Schizophrenien hatten weder häufigere $(t=0,962$, ns) noch schwerere Komplikationen $(t=1,462 ; n s)$ noch war der gewichtete Summenwert $(t=1,631$; ns) deutlich größer als bei den Gesunden. Patienten mit systematisch schizophrenen Krankheitsbildern hatten hingegen eine signifikant höhere Frequenz an Schwangerschafts- und Geburtskomplikationen als die unsystematischen Schizophrenen $(t=2,011 ; p<0,05)$ und als die gesunden Kontrollpersonen $(t=3,256 ; p<0,01)$.

Gegenüber Kontrollen war bei systematischen Schizophrenen der Schweregrad der Komplikationen ( $p<0,05$; $t=2,086)$ und auch der gewichtete Summenwert der Komplikationen $(p<0,01 ; t=2,792)$ signifikant erhöht.

Skala zur Erfassung von Geburtskomplikationen (Lewis \& Murray, 1987; Abb. 1)

In dieser Skala hatten $\mathbf{4 7}$ der 80 schizophrenen Patienten (58\%) mindestens eine sichere oder wahrscheinliche Schwangerschafts- oder Geburtskomplikation. Da bei 39 von 80 gesunden Probanden ( $49 \%$ ) die Mütter ebenfalls von Komplikationen berichteten, differierten beide Gruppen nicht. Abb. 1 zeigt, daß die Anzahl derer, die in der Anamnese pathologische Schwangerschafts- oder Geburtsvorkommnisse hatten, bei 

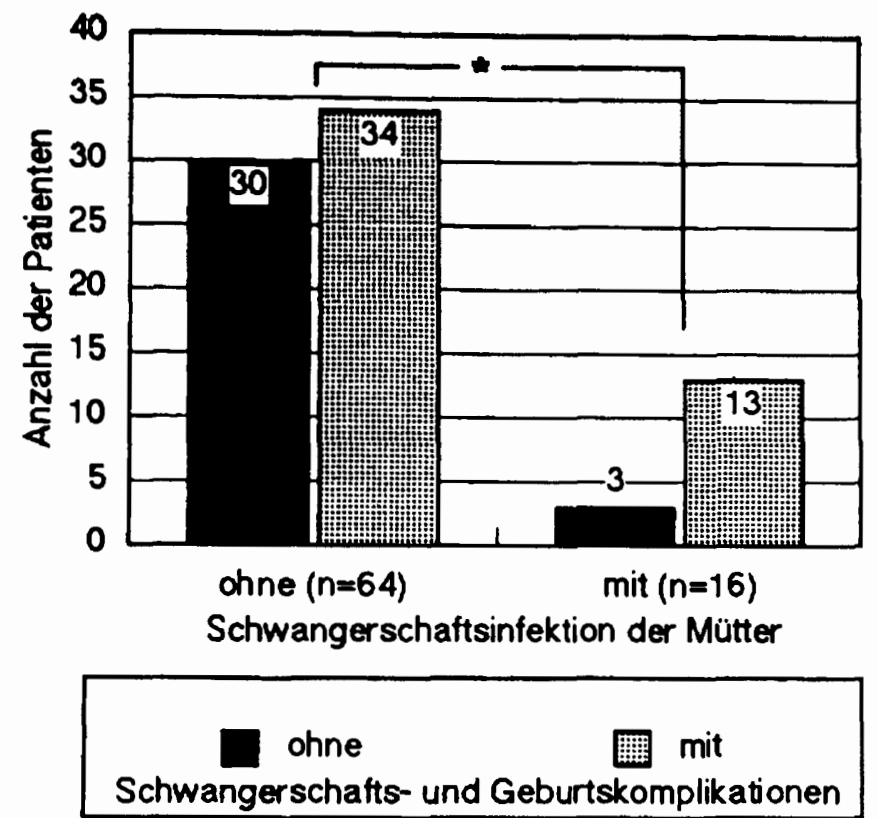

Abb. 2 Schwangerschaftsinfektionen und weitere perinatale Komplikationen. Mütter mit Schwangerschaftsinfektionen (hochsignifikant gehăuft bei systematischen Schizophrenien) hatten signifikant vermehrt zusătzliche obstetrische Komplikationen $(p<0,05$; $\mathrm{df}=1 ; \mathrm{Chi}_{2}=4,18$ ).

den systematischen Schizophrenen deutlich höher war als bei den Kontrollen $\left(\mathrm{Chi}_{2}=4,29 ; \mathrm{df}=1 ; \mathrm{p}<0,05\right)$ und bei den $\mathrm{Pa}$ tienten mit unsystematischen Schizophrenien $\left(\mathrm{Chi}_{2}=3,09 ; \mathrm{df}=\right.$ $1 ; \mathrm{p}<0,1)$.

Bei systematischen Schizophrenen standen Schwangerschafts- und vor allem Geburtskomplikationen in einem engen Zusammenhang mit dem Auftreten von Infektionserkrankungen in den Schwangerschaften ihrer Mütter. In der verwandten Skala von Lewis und Murray (1987) selbst werden Schwangerschaftsinfektionen nicht als Komplikationen berücksichtigt. 13 der 16 Mütter mit Schwangerschaftsinfektionen hatten in dieser Skala weitere pathologische Vorkommnisse in der Schwangerschaft und bei der Geburt (Abb. 2). Dies war signifikant $\left(\mathrm{Chi}_{2}=4,18 ; \mathrm{df}=1 ; \mathrm{p}<0,05\right)$ gegenüber den Müttern ohne Schwangerschaftskomplikationen.

Bei Patienten mit unsystematischen Schizophrenien, die insgesamt weniger geburtshilfliche Zwischenfälle durchgemacht hatten, bestand eine sehr enge Korrelation zwischen dem Auftreten von Komplikationen und dem Alter, in dem die Patienten erstmals stationär psychiatrisch behandelt wurden. Patienten mit komplizierten Schwangerschafts- und Geburtsverläufen waren mit 20,5 ( $\pm 4,92)$ Jahren signifikant früher hospitalisiert worden als diejenigen ohne Komplikationen mit 25,6 $( \pm 6,10)$ Jahren $(t=3,147 ; p<0,01)$. Im Median waren unsystematische Schizophrene mit 21 Jahren ersthospitalisiert worden. Diejenigen, die vor diesem Zeitpunkt erstmals stationär behandelt wurden, hatten mehr geburtshilfliche Komplikationen als diejenigen, die später hospitalisiert worden waren $\left(\mathrm{Chi}_{2}=4,45 ; \mathrm{df}=1 ; \mathrm{p}<0,05 ; \mathrm{Abb} .3 \mathrm{a}\right)$.

Bei systematischen Schizophrenen bestand keine Korrelation zwischen dem Auftreten von Schwangerschafts- und Geburtskomplikationen und dem Zeitpunkt der

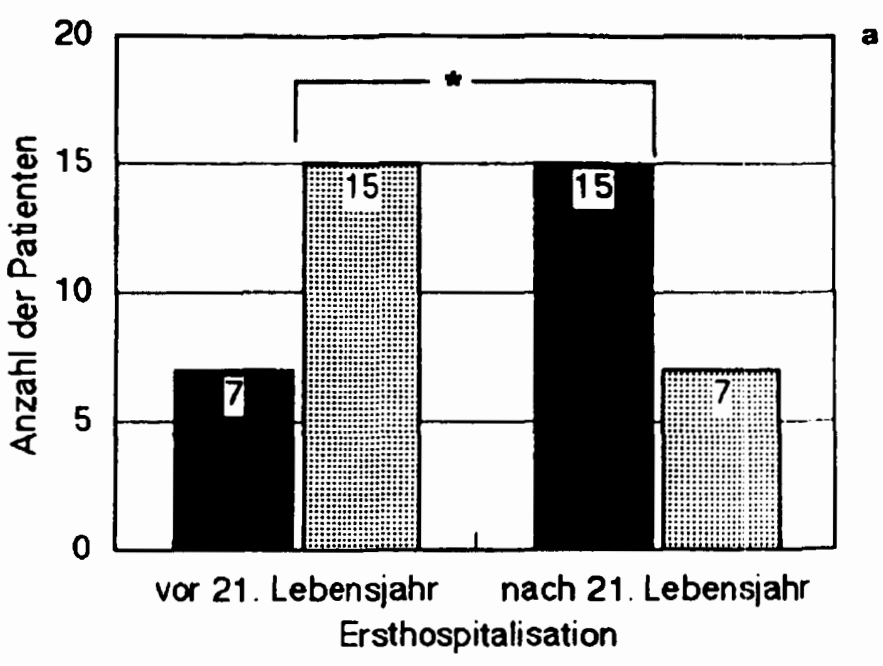

ohne

䧳 mit

Schwangerschafts- und Geburtskomplikationen

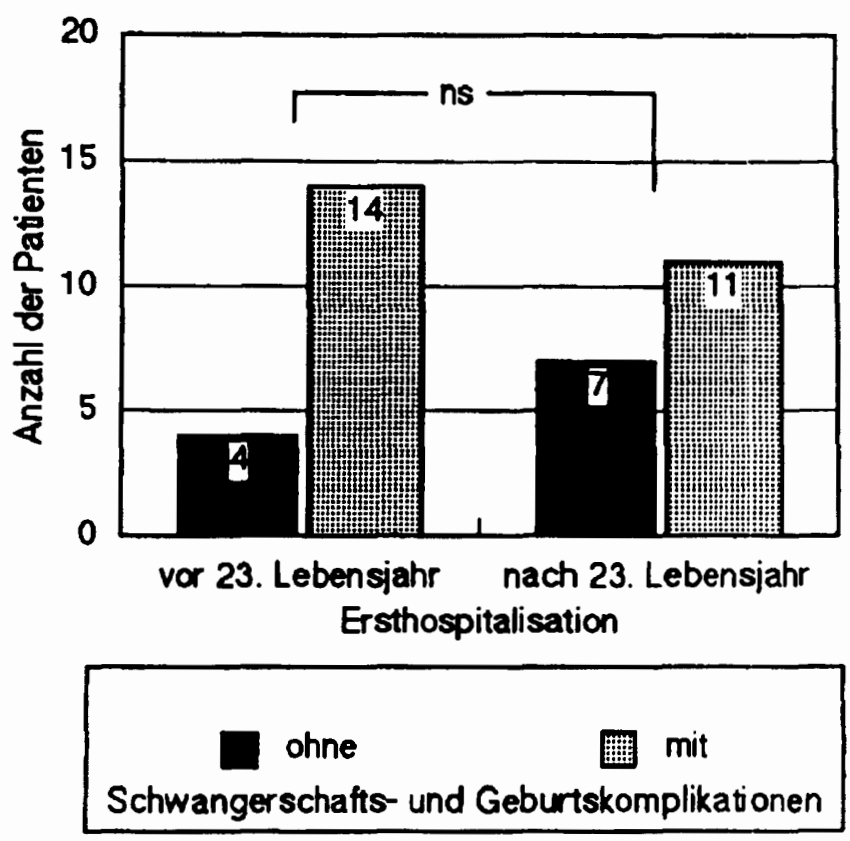

Abb.3 Schwangerschafts- und Geburtskomplikationen und Zeitpunkt der Ersthospitalisierung. Bei den unsystematischen Schizophrenen (Median des Alters bei Ersthospitalisierung: 21,5 Jahre) waren die vor dem 21. Lebensjahr Erkrankten siģnifikant häufiger von Komplikationen betroffen als die später hospitalisierten Patienten ( $p<0,05$; df = 1; Chiz = 4,18; Abb. 3a). Beil den systematischen Schizophrenen (Median des Alters bei Ersthospitalisierung: 23 Jahre) bestand kein Zusammenhang zwischen dem Auftreten von Komplikationen und dem Zeitpunkt der ersten stationären Behandlung (ns; $d f=1 ; C_{1} i_{2}=0,52 ; A b b .3 b$ ).

ersten stationären Aufnahme. Patienten mit Komplikationen wurden durchschnittlich im Alter von $23,0( \pm 8,48)$ Jahren, diejenigen ohne im Alter von 25,0 ( $\pm 6,87)$ Jahren hospitalisiert. Systematische Schizophrene waren im Median mit 23 Jahren erstmals stationär psychiatrisch behandelt worden. Komplikationen waren bei Patienten mit Ersthospitalisierung vor dem 23 . Lebensjahr nicht häufiger als bei den später Ersthospitalisierten $\left(\mathrm{Chi}_{2}=0,52 ; \mathrm{df}=1\right.$, ns; Abb. 3 b). 


\section{Diskussion}

Um Aufschluß zu erhalten über die Häufigkeit von Schwangerschafts- und Geburtskomplikationen bei schizophrenen Psychosen und über ihre Bedeutung für die Entstehung dieser Erkrankungen, führten wir eine retrospektive Untersuchung bei Müttern von chronisch schizophrenen Patienten und von alterskorrelierten gesunden Personen durch. Zur Wertung der Komplikationen verwendeten wir die Skalen von Parnas et al. (1982) und Lewis und Murray (1987). In diesen beiden Skalen fanden wir keinen Unterschied im Auftreten von Schwangerschafts- und Geburtskomplikationen zwischen gesunden Kontrollpersonen und der „Gruppe der Schizophrenien" als einer Krankheitseinheit. Diese Befunde stimmten überein mit einer Studie von Turner et al. (1986) bei schizophrenen Patienten, die innerhalb der ersten beiden Krankheitsjahre untersucht worden waren, und bei denen Verlaufskriterien unberücksichtigt geblieben waren (Tab. 1b). Auch Done et al. (1991) hatten in einer Geburtenkohorte von knapp 17000 Personen keine signifikante Erhöhung von Schwangerschaftskomplikationen und geburtstraumatischen Ereignissen bei den wenigen, die später wegen einer schizophrenen Erkrankung hospitalisiert wurden, gefunden.

In unsere Untersuchung eingeschlossen waren unsystematische und systematische Schizophrene nach Leonhard (1986), die sämtlich einen chronischen Verlauf und deutliche Residualzustände zeigten. Bei Leonhards systematischen Schizophrenien, die einen einförmig chronischen Verlauf mit einer fest umrissenen Symptomatologie zeigen, war die Häufigkeit von Störungen der prä- und perinatalen Zeit signifikant auf das Doppelte erhöht. Bereits $\mathrm{McNeil}$ und Kaij (1978) hatten darauf hingewiesen (Tab. 1 b), daß eng definierte „Prozeßschizophrene" eine signifikant höhere Zahl an Geburts-und perinatalen Störungen aufweisen als gesunde Kontrollen. Patienten mit gutartigen, remittierenden, schizophrenieähnlichen Psychosen, bei denen ebenfalls Schwangerschafts- und Geburtskomplikationen gehäuft sein kőnnen (McNeil, 1988; Jacobson und Kinney, 1980), waren in unsere Untersuchung nicht miteingeschlossen.

Unsystematische Schizophrenien nach Leonhard, die schubformig verlaufen und in unterschiedlich schwere Residuale ausmünden, unterscheiden sich nach unseren Befunden in bezug auf Häufigkeit und Schwere der Komplikationen nicht von gesunden Kontrollen. Bei diesen nach Leonhards Befunden überwiegend genetisch determinierten Schizophrenien waren Schwangerschafts- und Geburtskomplikationen jedoch sehr eng assoziiert mit einem frühen Erkrankungsbeginn (Abb. 3 a). Auch $\mathrm{De}$ Lisi et al. (1987) konnten zeigen, daß bei familiären Schizophrenien diejenigen mit Komplikationen wesentlich früher als diejenigen ohne Komplikationen hospitalisiert wurden. Bei systematischen Schizophrenen bestanden derartige Zusammenhänge nicht (Abb. 3b). Dies erklärt möglicherweise die unterschiedlichen Befunde früherer Untersucher (Tab. 3). Wilcox und Nasrallah (1987a) sowie Reddy et al. (1990) hatten keine Einflußnahme von prä- und perinatalen Störungen auf den Zeitpunkt der ersten Hospitalisierung gefunden. Andere (Pollack et al., 1966; Pearlson et al., 1985; Owen et al., 1988; $O^{\prime}$ Callaghan et al., 1992) fanden geburtshilfliche Zwischenfälle eng korreliert mit einer frühen Ersthospitalisierung.

Wenn innerhalb der modernen Klassifikationssysteme genetische Zuflüsse (erfaßt durch eine positive oder negative Familienanamnese für weitere schizophrene Erkrankungen) mit dem Auftreten von Schwangerschafts- und Geburtskomplikationen korreliert wurden, ergaben sich widersprüchliche Befunde (Tab. 2). Während Wilcox und Nasrallah (1987a), Schwarzkopf et al. (1988) sowie $O^{\prime}$ Callaghan et al. (1990a) berichteteten, daß Komplikationen bei Schizophrenen mit leerer Familienanamnese signifikant überwiegen, fanden andere (Pearlson et al., 1985; Nimgaonkar et al., 1988; Reddy et al., 1990) keine Assoziation von negativer Familienanamnese und obstetrischen Komplikationen. Nach unseren Befunden (Stöber et al., 1993 b) sind Geburts- und Schwangerschaftskomplikationen nur dann bei Patienten mit negativer Familienanamnese gehäuft, wenn diese gleichzeitig die diagnostischen Kriterien einer systematischen Schizophrenie nach Leonhard (1986) erfüllen. Bei den Patienten mit systematischen, nach Leonhards Befunden nicht hereditären Schizophrenien ist nicht nur die Anzahl derer, die pathologische Schwangerschaften und Geburten aufweisen, größer als bei den unsystematischen genetisch determinierten Schizophrenien (Abb. 1), sondern auch die Hăufigkeit und der Schweregrad der Komplikationen sind signifikant erhöht (Tab. 4).

Die systematischen Schizophrenien hatten eine hochsignifikante Häufung von Infektionserkrankungen der Mütter im mittleren Schwangerschaftsdrittel und insbesondere im 5. Schwangerschaftsmonat (Stöber et al., 1992; 1993c). Vor allem im 2. Trimenon verstärkt sich die Wanderung der Neurone von der ventrikulären Zone zu den vorbestimmten Positionen im Zentralnervensystem (Rakic, 1988). Eine Unterbrechung der neuronalen Migration zu diesem Zeitpunkt scheint mitursächlich zu sein für die von Jakob und Beckmann (1986) gefundenen, eng umschriebenen zytoarchitektonischen Veränderungen im Gyrus parahippocampalis bei Post-mortem-Untersuchungen an Gehimen Schizophrener (bestätigt von: Falkai et al,, 1988; Arnold et al., 1991 sowic Beckmann u. Jakob, 1991). Insbesondere bei den Schizophrenen, deren Mütter eine Schwangerschaftsinfektion durchgemacht hatten, kamen weitere pathologische Vorkommnisse während der Gestationszeit und während der Geburt hinzu. Schwere Geburtskomplikationen weisen auf Störungen des zerebralen Metabolismus, hypoxisch-ischämische Schädigungen und auf neuronale Zelluntergänge hin (Hill. 1991; Gluckman u. Williams, 1992). Asphyxiebedingte zerebrale Ischämien können hier zu intrazellulärem Ödem und rascher Zellyse oder zu einem verzögerten Zelltod durch Veränderungen des neuromodulatorischen Milieus (Hagberg et al., 1987) oder durch strukturelle Desorganisationen (Jankowsky, 1986; Raff. 1992) führen.

Zusammengefaßt sind Schwangerschafts- und Geburtskomplikationen bei den unsystematischen genetisch determinierten Schizophrenien nicht gehäuft, prädisponieren aber bei diesen Psychosen zu einem frühen Erkrankungsbeginn. Präund perinatale Störungen sind bei systematischen Schizophrenien hingegen gehäuft. Schwangerschaftsinfektionen der Mütter im 2. Trimenon und zusätzliche perinatale Komplikationen scheinen wichtige Faktoren zu sein für die Entstehung dieser nicht hereditären Schizophrenien. 


\section{Literatur}

1 Arnold, S. E., B. T. Hyman, G. W. van Hoesen, A. R. Damasio: Cytoarchitectural abnormalities of the entorhinal cortex in schizophrenia. Arch. Gen. Psychiatry 48 (1991) 625-632

2 Astrup, C.: The chronic schizophrenias. Universitätsforlaget, Oslo 1979

3 Beckmann, $H$., H. Jakob: Prenatal disturbances of nerve cell migration in the entorhinal region: a common vulnerability factor in functional psychoses? J. Neural. Transm. (Gen. Sect.) 84 (1991) 155 $-164$

4 DeLisi, L. E., L. R. Goldin, M. E. Maxwell, D. M. Kazuba, E. S. Gershon: Clinical features of illness in siblings with schizophrenia or schizoaffective disorder. Arch. gen. Psychiatry 44 (1987) 891-896

${ }^{5}$ DeLisi, L. E., L. R. Goldin, J. R. Hamovit, M. E. Marwell. D. Kurtz, $E$. S. Gershon: A family study of the association of increased ventricular size with schizophrenia. Arch. Gen. Psychiatry 43 (1986) $148-153$

6 Done, D. J., E. C. Johnstone, C. D. Frith, J. Golding, P. M. Shepherd, $T$. $J$. Crow: Complications of pregnancy and delivery in relation to psychosis in adult life: data from the British perinatal mortality survey sample. Br. Med. J. 302 (1991) 1576-1581

7 Eagles, J. M., I. Gibson, M. H. Bremner, F. Clunie, K. P. Ebmeier, N. C. Smith: Obstetric complications in DSM-1II schizophrenics and their siblings. Lancet 335 (1990) $1139-1141$

B Falkai, P., B. Bogerts, M. Rozumek: Limbic pathology in schizophrenia: The entorhinal region - a morphometric study. Biol. Psychiatry 24 (1988) 515-521

9 Franzek, E., H. Beckmann: Syndrom- und Symptomentwicklung schizophrener Langzeitverläufe. Nervenarzt 62 (1991) 549-556

10 Franzek, E., H. Beckmann: Schizophrenia: Not a disease entity? A study of 57 longterm hospitalized chronic schizophrenics. Europ. J. Psychiatry 6 (1992) 97-108

11 Gluckman, P. D., C. E. Williams: When and why do brain cells die? Dev. Med. Child Neurol. 34 (1992) 1010-1021

12 Goodman, R.: Are complications of pregnancy and birth causes of schizophrenia? Dev. Med. Child Neurol. 30 (1988) 391-395

13 Hagberg, H., P. Andersson, I. Kjellmer, K. Thiringer, M. Thordstein: Extracellular overflow of glutamat, aspartate, GABA and taurine in the cortex and basal ganglia of fetal lambs during hypoxia-ischemia. Neuroscience Letters 78 (1987) $311-317$

14 Hill, A.: Current concepts of hypoxic-ischemic cerebral injury in the term newborn. Pediatric Neurol. 7 (1991) 317-325

15 Jakob, H., H. Beclomann: Prenatal developmental disturbances in the limbic allocortex in schizophrenia. J. Neural. Transm. 65 (1986) 303 $-326$

16 Jacobson, B., D. K. Kinney: Perinatal complications in adopted schizophrenics and their controls: preliminary results. Acta Psychiatr. Scand. Suppl. 285 (1980) 337-348

17 Janowsky, J. S. B. L. Finlay: The outcome of perinatal brain damage: the role of normal neuron loss and axon retraction. Develop. Med. Child Neurol. 28 (1986) 375-389

IB Joffe, M., J. A. Grisso: Comparison of ante-natal hospital records with retrospective interviewing. J. Biol. Soc. Sci. 17 (1985) $113-119$

19 Kety, S. S., D. Rosenthal, P. H. Wender, F Schulsinger: The types and prevalence of mental illness in the biological and adoptive families of adopted schizophrenics. In: Kety, S. S., D. Rosenthal (eds.): The transmission of schizophrenia. Pergamon, Oxford (1968) 345-362

20 Lane, E. A., G. W. Albee: Comparative birth weights of schizophrenics and their siblings. J. Psychology 64 (1966) 227 - 231

${ }^{21}$ Leonhard, K.: Aufeilung der endogenen Psychosen in der Forschungsrichtung von Leonhard und Kleist. In: Kisker, $\mathcal{K} . P, J$. E. Meyer, M. Müller, E. Strömgren (Hrsg.): Psychiatrie der Gegenwart. Bd. II.1. Springer, Berlin, Heidelberg, New York (1972) 183-212

22 Leonhard, $K$.: Ein dominanter und ein rezessiver Erbgang bei zwei verschiedenen Formen der Schizophrenic. Nervenarzt 46 (1975) $242-248$

23 Leonhard, $K$.: Über erblich bedingte und psychosozial bedingte Schizophrenien. Psychiat. Ncurol. med. Psychol. 31 (1979) $606-626$
24 Leonhard, K.: Die Aufteilung der endogenen Psychosen und ihre differenzierte Ätiologie. Akademie-Verlag, Berlin 1986

25 Lewis, S. W., R. M. Murray: Obstetric complications, neurodevelopmental deviance, and risk of schizophrenia. J. Psychiatr. Res. 21 (1987) 413-421

26 Lewis, S. W. M. J. Owen, R. M. Murray: Obstetric complications and schizophrenia. In: Schulz, S. C., C. A. Tammina (Hrsg.): Schizophrenia: Scientific Progress. Oxford University Press, New York (1989) 56-68

27 Marcus, J., J. Auerbach, L. Wilkinson, C. M. Burack: Infants at risk for schizophrenia. The Jerusalem infant development study. Arch. Gen. Psychiatry 38 (1981) 703-713

$28 \mathrm{McNeil}$, T. F: Perinatal influences in the development of schizophrenia. In: Helmchen, H., F. A. Henn (Hrsg.): Biological perspectives of schizophrenia. Wiley, Chichester (1987) 125- 138

29 McNeil, T. F: Obstetric factors and perinatal injuries. In: Tsuang, M. T., J. C. Simpson (eds): Handbook of schizophrenia. Vol. 3: Nosology, Epidemiology and Genetics, Elsevier, Amsterdam, New York, Oxford (1988) 319-344

30 McNeil, T. F. L. Kaij: Obstetric factors in the development of schizophrenia: complications in the birth of preschizophrenics and in reproduction by schizophrenic parents. In: Wynne, L. C., R. L. Cromwell, S. Matthysse (eds.): The nature of schizophrenia. Wiley, New York (1978) 401-429

31 Molfese, V.J., B. Thomson: Optimality versus complications: assessing predictive values of perinatal scales. Child Dev. 56 (1985) 810-823

32 Nimgaonkar, V. L., S. Wessely, R. M. Murnay: Prevalence of familiality obstetric complications, and structural brain damage in schizophrenic patients. Br. J. Psychiatry 153 (1988) 191-197

$33 O^{\prime}$ Callaghan, E., C. Larkin, A. Kinsella, J. L. Waddington: Obstetrity complications, the putative familial-sporadic distinction, and tardive dyskinesia in schizophrenia. Br. J. Psychiatry 157 (1990) 578 -- 584

34 O'Callaghan, E., C. Larkin, J. L. Waddington: Obstetric cornplications in schizophrenia and the validity of matemal recall. Psychol. Med. 20 (1990) 89-94

35 O'Callaghan, E., T. Gibson, H. a. Colohan, P. Buckley, D. G. Walshe, C. Larkin, J. L. Waddington: Risk of schizophrenia in adults born after obstetric complications and their association with early onset of illness: a controlled study. Br. Med. J. 305 (1992) 1256-1259

36 Owen, M. O., S. H. Lewis, R. M. Murray: Obstetric complications and schizophrenia: a computed tomographic study. Psychol. Med. 18 (1988) $331-339$

37 Parnas, J., F. Schulsinger, T. W. Teasdale, H. Schulsinger, P. M. Feldman, $S$. A. Mednick: Perinatal complications and clinical outcome within the schizophrenia spectrum. Br. J. Psychintry 140 (1982) $416-420$

38 Peartson, G. D. D. J, Garbacz, P. J. Moberg H. S. Ahn, J. R. de Paulo: Symptomatic, familial, perinatal, and social correlites of computerized axial tomography (CAT) changes in schizoplurenics and bipolars. J. Nerv. Ment. Dis. 173 (1985) $42-50$

39 Polluck, M. I. M. Greenberg: Paranatal complications in hospitalized schizophrenic and nonschizophrenic patients. J. Hillside Hosp. 15 (1986) $191-204$

40 Pollack M. M. G. Woerner, W. Goodman, I. M. Greenberg: Childhood development patterns of hospitalized adult schizophrenic and nonschizophrenic patients and their siblings. Am. J. Orthopsychiatry 36 (1966) 510-517

41 Raff, M. C.: Social controls on cell survival and cell death. Nature 356 (1992) $397-400$

42 Rakic, P.: Specification of cerebral areas. Science 421 (1988) $170-176$

${ }^{43}$ Reddy, R., S. Mukherjee, D. B. Schnur, J. Chin, G. Degreef: History of obstetric complications, family history, and CT-scan findings in schizophrenic patients. Schizophrenia Research 3 (1990) 311314

44 Rieder, $R$. $O$., $D$. Rosenthal, $P$. Wender, $H$. Blumenthal: The offspring of schizophrenics. Fetal and neonatal deaths. Arch. Gen. Psychiatry 32 (1975) 200-211

45 Schwarzkopf, S. B., H. A. Nasrallah, S. C. Olson, J. A. Coffman, J. A. McLaughlin: Perinatal complications and genetic loading in schizophrenia: preliminary findings. Psychiatry Research 27 (1989) 233-239 
46 Stöber, G., E. Franzek, H. Beckmann: The role of maternal infectious diseases during pregnancy in the etiology of schizophrenia in the offspring. Eur. Psychiatry 7 (1992) 147-152

47 Stöber, G., H. Beckmann, G. Jungkunz, E. Franzek: Die „Proskinetische Katatonie" - Ein kasuistischer Beitrag zur Psychopathologie chronisch schizophrener Psychosen. Krankenhauspsychiatrie 4 (1993) $70-73$

48 Stöber, G., E. Franzek, H. Beckmann: Obstetric complications in distinct schizophrenic subgroups. Eur. Psychiatry (im Druck)

49 Stöber, G., H. Beckmann, E. Franzek: Schwangerschaftsinfektionen bei Müttern von chronisch Schizophrenen. Nervenarzt (im Druck)

so Tilley, B. C., A. B. Barmes, E. Bergstrath, D. Labarthe, K. L. Noller. $T$. Colton, E. Adam: A comparison of pregnancy history recall and medical records: implications for retrospective studies. Am. J. Epidemiology 121 (1985) 269-281

51 Turner, S. W. K. B. Toone, J. R. Brett-Jones: Computerized tomographic scan changes in early schizophrenia - preliminary findings. Psychol. Med. 16 (1986) 219-225

32 Wilcox, J. A., H. A. Nasrallah: Perinatal distress and prognosis of psychotic illness. Neuropsychobiology 17 (1987) 173175

33 Wilcox, J. A., H. A. Nasrallah: Perinatal insult as a risk factor in paranoid and nonparanoid schizophrenia. Psychopathology 20 (1987) 285-287

34 Woerner, M. G., M. Pollack. D. F. Klein: Pregnancy and birth complications in psychiatric patients: a comparison of schizophrcnic and personality disorder patients with their siblings. Acta Psychiatr. Scand. 49 (1973) $712-721$
Dr. med. G. Stöber

Psychiatrische Klinik und Poliklinik

der Universität Würzburg

Füchsleinstr. 15

97080 Würzburg 